AperTO - Archivio Istituzionale Open Access dell'Università di Torino

\title{
Targeting Metabolism to Counteract Tumor Angiogenesis: A Review of Patent Literature
}

\section{This is a pre print version of the following article:}

Original Citation:

\section{Availability:}

This version is available http://hdl.handle.net/2318/1669212

since 2019-02-18T10:27:04Z

Published version:

DOI:10.2174/1574892813666180528105023

Terms of use:

Open Access

Anyone can freely access the full text of works made available as "Open Access". Works made available under a Creative Commons license can be used according to the terms and conditions of said license. Use of all other works requires consent of the right holder (author or publisher) if not exempted from copyright protection by the applicable law. 
Targeting Metabolism to Counteract Tumor Angiogenesis: A Review of Patent

\section{Literature}

Petrillo S. ${ }^{1}$, Tolosano E. ${ }^{1}$, Munaron L. ${ }^{2}$, Genova T. ${ }^{2,3}$

Affiliations

1 Department of Molecular Biotechnology and Health Sciences, Molecular Biotechnology Center, University of Torino, Torino, Italy

2 Department of Life Sciences and Systems Biology, University of Torino, Torino, Italy

3 CIR Dental School, Department of Surgical Sciences, University of Torino, Torino, Italy

Keywords: Tumor angiogenesis, Vessel normalization, Endothelial cell metabolism, Angiogenesis inhibition

\section{Corresponding author:}

Tullio Genova, PhD

Department of Life Sciences and Systems Biology

Via Accademia Albertina, 13

10123 Torino, Italy

Phone: +39-011-6704663

e-mail: tullio.genova@unito.it 


\section{ABSTRACT}

Massive vessel recruitment is required to sustain rapid tumor growth by delivering oxygen and nutrients. Current strategies to counteract angiogenesis are mostly aimed at reducing tumor vessel density. However, many of these drugs have been shown to trigger hypoxia, thus exacerbating tumor aggressiveness. Promising results come from a completely different approach based on the "normalization" of the endothelial layer and the consequent improvement of the vascular function. Since endothelial metabolism has proved essential in the regulation of the angiogenic switch, many recent patents focus on agents able to inhibit specific metabolic pathways in tumor-associated endothelial cells (TECs) in order to provide vessel normalization. This new strategy would ameliorate drug delivery to the tumor meanwhile reducing invasiveness and metastatisation. These findings might have important implication in clinics and could be particularly relevant to patients developing resistance to traditional anti-angiogenic drugs. 


\section{INTRODUCTION}

\section{The angiogenic process}

New blood vessels are formed from pre-existing ones through the angiogenic process which involves migration, proliferation and differentiation of endothelial cells (ECs) lining the blood vessels wall. ECs in the adult organism are mostly quiescent but retain the ability to rapidly activate in response to growth factors such as the vascular endothelial growth factor (VEGF) or under hypoxic condition and switch towards an angiogenic state(1). In accordance with the model of vascular sprouting, in the presence of pro-angiogenic stimuli, ECs differentiate into specialized subtypes. In particular, "tip" cells feel the pro-angiogenic cues and guide the new sprout thanks to the presence of several motile structures as filopodia and lamellipodia, which allow directional locomotion. Meanwhile, "stalk" cells proliferate to elongate the sprout and to form a vascular lumen. When the new branch is established and properly perfused, ECs acquire again the quiescent "phalanx" phenotype.

Importantly, ECs needs to adapt their metabolic profile when switching from quiescence to vascular branching $(1,2)$. In the recent years, growing evidences highlighted the pivotal role of endothelial metabolism in controlling angiogenesis(3-6). Despite their direct access to blood oxygen, ECs preferentially rely on glycolysis for energy supply, with most glucose entering the cell converted in lactate(1). This metabolic feature allows ECs to face the high-to-low oxygen availability cycles deriving from the continuous vessel remodelling. Notably, the glycolytic flux is further enhanced upon induction of the angiogenic switch in order to increase the production of adenosine triphosphate (ATP) and macromolecules for cell division(7). Interestingly, glycolytic ATP production has been shown to compartmentalize within the motile cytoplasmic projections of "tip" cells where it has to rapidly provide energy for cytoskeletal remodelling during migration(7).

Several papers have recently pointed out the critical and direct involvement of glycolysis and fatty acid oxidation (FAO) in the regulation of most endothelial functions(8). For instance, genetic or pharmacological inactivation of the glycolytic activator phosphofructokinase-2/fructose-2,6-bisphosphatase-3 (PFKFB3) leads 
to impaired endothelial proliferation and migration as well as reduced vascular branching both in vitro and in vivo(7). Conversely, inhibition of mitochondrial respiration has been shown not to affect angiogenesis(7). More than $85 \%$ of glucose entering ECs is then converted into lactate(7). Importantly, lactate has a proangiogenic role by increasing VEGF production in ECs through stabilization of hypoxia-inducible transcription factor-1 $\alpha$ (HIF-1 $\alpha)$ and promoting cell migration $(9,10)$. The FAO pathway has proved essential for angiogenesis too. Indeed, endothelial loss of the mitochondrial importer of fatty acids (FAs), i.e. CPT1a, reduces cell proliferation in vitro and affects retinal vascular development in mice(11). This phenotype is likely caused by depletion of the precursors for nucleotide biosynthesis, which is turn required for DNA replication and cell proliferation. Glutamine is a key amino acid involved in ECs metabolism(12). Firstly, glutaminolysis is an important analplerotic pathway of the Krebs cycle by replenishing the cellular pool of $\alpha$ ketoglutarate. Furthermore, glutamine is an important source of carbons for protein synthesis as well as a precursor of glutathione (GSH), a key molecule involved in cell redox homeostasis. Notably, glutamine deprivation in ECs results in impaired proliferation and reduced vessel sprouting in vitro(13). Moreover, genetic ablation of glutaminase-1 (GLS1), the enzyme responsible for glutamine conversion into glutamate, causes vascular defects in the retina of 5-days pups(13). These indications, taken together, widely demonstrate that specific metabolic pathways regulate the angiogenic response in ECs.

\section{Tumor angiogenesis}

Blood vessels deliver oxygen and nutrients to all tissues, including tumors. Excessive and deregulated angiogenesis is an important hallmark of cancer progression. To sustain rapid growth, cancer cells secrete in the tumor microenvironment high levels of pro-angiogenic factors as vascular endothelial growth factor-A (VEGF-A), placental growth factor (PIGF) and basic fibroblast growth factor (bFGF). The exposure of tumor ECs to imbalanced growth factor signals promotes vessel overgrowth(14). However, in a counterintuitive manner, this aberrant angiogenesis leads to the formation of a non-functional vasculature. Indeed, tumor vessels appear highly disorganized, tortuous and dilated $(15,16)$. Moreover, the loose association with mural cells results in excessive permeability, poor perfusion and hypoxia(16). These structural and functional alterations eventually promote cancer cell metastatisation and limit drugs delivery to the tumor. For these 
reasons and since blood vessels are essential to sustain tumor growth, strategies to inhibit tumor angiogenesis have been developed to treat cancer.

\section{Therapeutic strategies - canonical approaches}

As a master regulator of angiogenesis, the VEGF pathway is a main target of anti-angiogenic therapies. In particular, among the molecules that are approved and/or in clinical development, there are monoclonal antibodies (mAb) against VEGF-A (i.e. Bevacizumab) or its receptor (VEGFR2) (i.e. Ramucirumab) and small molecules able to block receptor tyrosine kinases (RTKs) as VEGF and PDGF receptors (e.g. Sunitinib, Sorafenib). Another strategy to inhibit tumor angiogenesis focuses on the block of Angiopoietin-2 (ANG2), one of the ligands of the Tie2 receptor (i.e. AGM386)(17).

Anti-angiogenic therapies are currently providing survival benefits to many cancer patients, in particular in combination with chemotherapy. However, these therapies have been shown to only slightly improve the overall survival. The most important reason why most anti-angiogenic drugs failed to pass the clinical trial phase relies on the radical difference between normal and tumor ECs. For instance, it has been recently demonstrated that, unlike normal ECs (NECs), endothelial cells isolated from prostate tumors show a reduced sensitivity in term of proliferation, survival and motility to the oral non-selective tyrosine kinase inhibitor Sunitinib(18). Moreover, ECs behaviour is widely influenced by the specific stimuli associated to the tumour micro-environment and some important mechanisms of cell migration observed in NECs are not active in TECs(19-22). In conclusion, the important functional differences between NECs and TECs together with the onset of resistance mechanisms and the side effects associated to canonical anti-angiogenic treatments highlighted the need for new molecular targets as well as novel approaches.

\section{Therapeutic strategies - Tumor vessel normalization}

A different strategy, based on the concept of tumor vascular normalization, is aimed at improving the vascular function instead of destroying vessels. This effect involves the attenuation of hyper-permeability, the increase in pericytes coverage and the restoration of perfusion(23). In combination with chemotherapy, endothelial normalization therefore enhances drug delivery and prevents the induction of hypoxia linked to 
the drastic block of tumor's blood supply, likely limiting the risk of metastasis formation(14). Importantly, some anti-angiogenic agents as Bevacizumab, have been shown to give just a partial reduction of vessel density when administrated at low doses, therefore promoting an overall normalization of the vasculature with numerous advantages(24).

In addition to addressing the VEGF / VEGFR and ANG / TIE-2 axes, vascular normalization can be achieved also by targeting ECs metabolism. Indeed, the metabolic switch associated to the angiogenic response of tumor ECs has recently revealed to be a new promising therapeutic target. For instance, a partial inhibition of glycolysis has already proved effective in counteracting tumor angiogenesis in vivo by promoting vessel normalization. In particular, PFKFB3 haplo-deficiency in ECs results in a better-functioning tumor vasculature, thus leading to reduced metastatisation and improved delivery of chemotherapeutics to the tumor(25). Similar results can be obtained also through a pharmacological approach, by injecting the PFKFB3-blocker 3(3-pyridinyl)-1-(4-pyridinyl)-2-propen-1-one (3PO) at low doses in mice(25). Consistently, a recent patent provides siRNAs directed against PFKFB3 for the treatment of pathological angiogenesis. This patent concerns not only the treatment of cancer but involves also applications for the treatment of pathological angiogenesis such as for example macular degeneration(26). Other patents (not specifically directed against tumor vessels) are based on a PFKFB3 inhibitor in combination with an immune checkpoint inhibitor to treat cancer and to stimulate anti-tumor immunity(27).

An alternative way to counteract pathological angiogenesis by targeting ECs metabolism is based on the inhibition of FAO. Indeed, interfering with the FAO rate-limiting enzyme CPT1a, impairs endothelial sprouting without affecting cell migration(11). Even if targeting CPT1a was shown effective in treating pathological angiogenesis conditions such as age-related macular degeneration, diabetic retinopathy, diabetic maculopathy and proliferative retinopathies, this strategy may not be suitable to the tumor context. Indeed, CPT1a silencing has been shown to induce hyper-permeability of ECs monolayers in vitro and leakage of blood vessels in vivo(28). Patents from Carmeliet and Shoors indeed provides both siRNAs and inhibitors directed against CPT1a, which have proven effective for the treatment of pathological angiogenesis different from the tumor context(29-32). 
Cancer cells need to reprogram their metabolism to sustain high division rates and to survive under hypoxic conditions. In particular, they switch their metabolism towards aerobic glycolysis, the so-called Warburg effect, to fulfil the growing tumor energy demands(33). Because of this substantial increase in the glycolytic flux, high levels of lactate are found in the tumor microenvironment, which contribute to acidosis. As stated above, the glycolytic product lactate drives angiogenesis in ECs through HIF-1 $\alpha$ stabilization. Hence, the lactate secreted by cancer cells has a paracrine effect on ECs and works as a signalling molecule aimed at increasing angiogenesis. Consistently, high levels of lactate in human cancer correlates with the invasiveness and poor prognosis of the disease(34). Targeting the endothelial lactate importer, i.e. monocarboxylate transporter 1 (MCT1), actually results in reduced tumor angiogenesis(35). To date, patents aimed at inhibiting MCT1 are only related to the treatment or diagnosis of some type of cancer(36). Anyway, in the future, a dual effect targeting both cancer cells and tumor-associated ECs could bring important advantages.

Glutaminolysis is enhanced in both tumor-associated ECs and cancer cells. Interestingly, the inhibition of glutamine synthetase (GS) strongly affects vascular branching, in both developmental and pathological angiogenesis. Agents targeting GS can be used for the treatment of diseases characterized by pathological angiogenesis such as macular degeneration. In particular, a specific patent concerns both siRNAs and specific inhibitors aimed at reducing the activity of the GS enzyme(37).

Another in vivo study showed that a decrease in the activity of the oxygen-sensor protein PHD2 induces endothelial normalization thus suppressing tumor invasiveness and metastasis(23, 38). In this model, vessel normalization is due to a shift from "tip" towards "phalanx" morphology of tumor-associated ECs, which helps to restore a normal endothelial layer. The endothelial re-adaptation is therefore responsible for improving perfusion and oxygenation so that the resulting tumor appears less glycolytic and aggressive(38, 39). A patent of 2014 refers these beneficial outcomes due to the administration a PHD2 inhibitor wherein the inhibitory effect is achieved at the DNA or RNA level(40-45).

Other promising results come from studies on the anti-malarian drug chloroquine. This agent acts as an autophagy inhibitor and shows anti-cancer activity at high doses. Conversely, a low dose of chloroquine is able to induce a more quiescent phenotype in tumor ECs therefore promoting vessel normalization via 
autophagy-independent mechanisms $(46,47)$. A related patent shows a method to treat cancer by using a pharmaceutical composition of an anti-angiogenic drug (i.e. Avastin) together with the autophagy inhibitor chloroquine(48).

Taken together these data highlighted the pivotal role of endothelial metabolism in driving angiogenesis and investigated different strategies to target specific metabolic pathways to treat cancer.

\section{CONCLUSIONS}

The present work reviews the recent patents on strategies to counteract tumor vascularization. In particular, great efforts have been made to elucidate the role of endothelial metabolism in the control of angiogenesis in order to open new windows of therapeutic intervention. Promising results come from studies on strategies aimed at inhibiting the metabolic pathways involved in the endothelial angiogenic response such as glycolysis, FAO and glutaminolysis. The difficulty of translating these findings from basic science to the clinical use still places a great challenge and requires further investigation. 
Figure 1. Endothelial metabolic targets to counteract tumor angiogenesis. GS, Glutamine Synthetase; CPT1a, Carnitine palmitoyltransferase I; MCT1, monocarboxylate transporter 1; 3PO, specific inhibitor of the glycolytic regulator PFKFB3; a-KG, alpha-Ketoglutaric acid; OAA, Oxaloacetic acid; TCA, tricarboxylic acid cycle; Glut1, Glucose transporter 1; 3PG, 3-Phosphoglyceric acid; PEP, Phosphoenolpyruvate; Ac-CoA, Acetyl coenzyme A; FAO, Fatty acid oxidation.

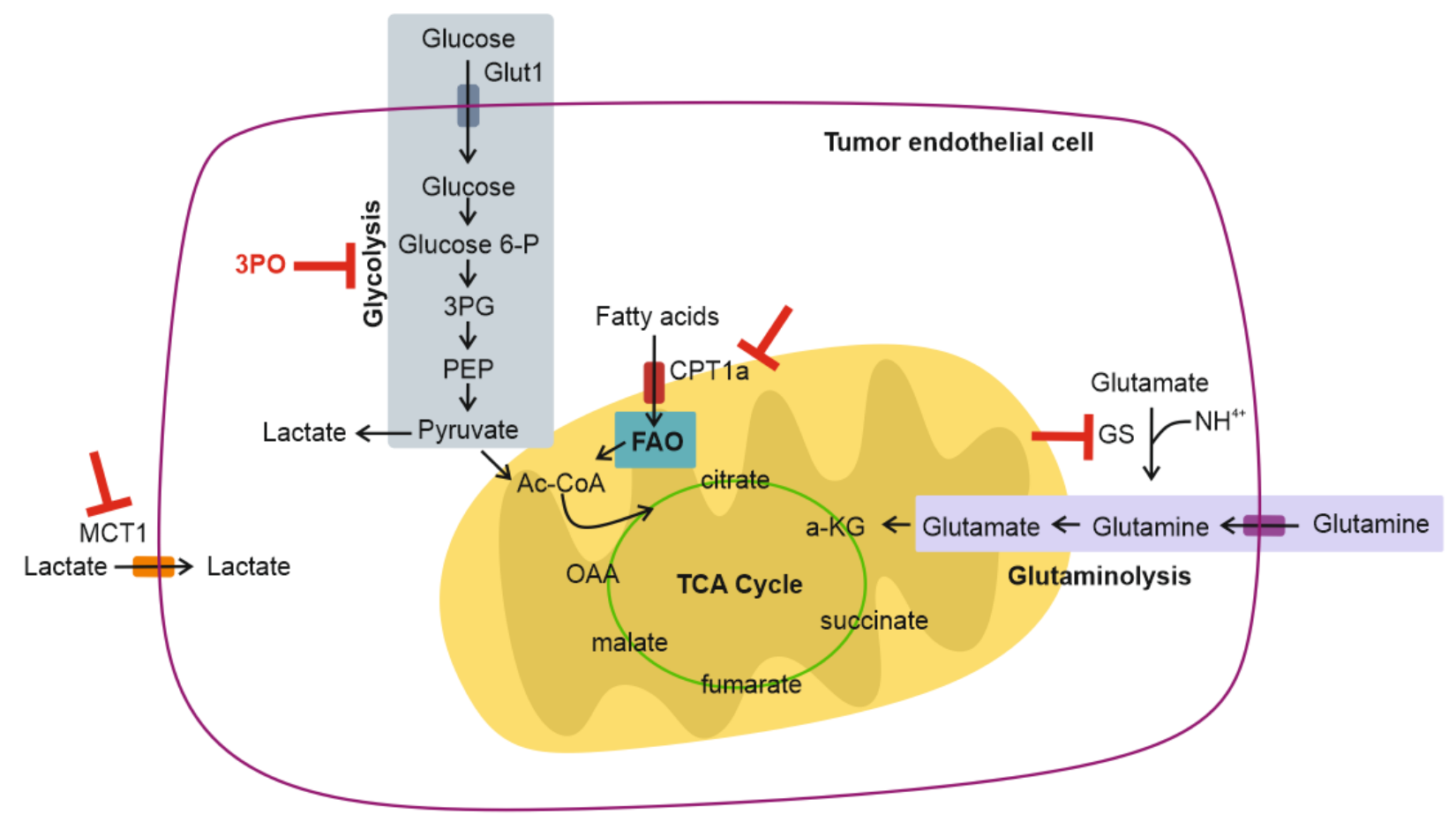




\section{References}

1. De Bock K, Georgiadou M, Carmeliet P. Role of endothelial cell metabolism in vessel sprouting. Cell Metab. 2013;18(5):634-47.

2. Uebelhoer M, Iruela-Arispe ML. Cross-talk between signaling and metabolism in the vasculature. Vascul Pharmacol. 2016;83:4-9.

3. Vandekeere S, Dewerchin M, Carmeliet P. Angiogenesis Revisited: An Overlooked Role of Endothelial Cell Metabolism in Vessel Sprouting. Microcirculation. 2015;22(7):509-17.

4. Draoui N, de Zeeuw P, Carmeliet P. Angiogenesis revisited from a metabolic perspective: role and therapeutic implications of endothelial cell metabolism. Open Biol. 2017;7(12).

5. Teuwen LA, Draoui N, Dubois C, Carmeliet P. Endothelial cell metabolism: an update anno 2017. Curr Opin Hematol. 2017;24(3):240-7.

6. Petrillo S, Chiabrando D, Genova T, Fiorito V, Ingoglia G, Vinchi F, et al. Heme accumulation in endothelial cells impairs angiogenesis by triggering paraptosis. Cell Death Differ. 2017.

7. De Bock K, Georgiadou M, Schoors S, Kuchnio A, Wong BW, Cantelmo AR, et al. Role of PFKFB3-driven glycolysis in vessel sprouting. Cell. 2013;154(3):651-63.

8. Cantelmo AR, Brajic A, Carmeliet P. Endothelial Metabolism Driving Angiogenesis: Emerging Concepts and Principles. Cancer J. 2015;21(4):244-9.

9. Beckert S, Farrahi F, Aslam RS, Scheuenstuhl H, Königsrainer A, Hussain MZ, et al. Lactate stimulates endothelial cell migration. Wound Repair Regen. 2006;14(3):321-4.

10. Hunt TK, Aslam RS, Beckert S, Wagner S, Ghani QP, Hussain MZ, et al. Aerobically derived lactate stimulates revascularization and tissue repair via redox mechanisms. Antioxid Redox Signal. 2007;9(8):111524.

11. Schoors S, Bruning U, Missiaen R, Queiroz KC, Borgers G, Elia I, et al. Fatty acid carbon is essential for dNTP synthesis in endothelial cells. Nature. 2015;520(7546):192-7.

12. Teuwen LA, Geldhof V, Carmeliet P. How glucose, glutamine and fatty acid metabolism shape blood and lymph vessel development. Dev Biol. 2017.

13. Huang $\mathrm{H}$, Vandekeere $\mathrm{S}$, Kalucka J, Bierhans $\mathrm{L}$, Zecchin $\mathrm{A}$, Brüning $\mathrm{U}$, et al. Role of glutamine and interlinked asparagine metabolism in vessel formation. EMBO J. 2017;36(16):2334-52.

14. Wong PP, Bodrug N, Hodivala-Dilke KM. Exploring Novel Methods for Modulating Tumor Blood Vessels in Cancer Treatment. Curr Biol. 2016;26(21):R1161-R6.

15. Cantelmo AR, Pircher A, Kalucka J, Carmeliet P. Vessel pruning or healing: endothelial metabolism as a novel target? Expert Opin Ther Targets. 2017;21(3):239-47.

16. Viallard C, Larrivée B. Tumor angiogenesis and vascular normalization: alternative therapeutic targets. Angiogenesis. 2017;20(4):409-26.

17. Singh $\mathrm{M}$, Ferrara N. Modeling and predicting clinical efficacy for drugs targeting the tumor milieu. Nat Biotechnol. 2012;30(7):648-57.

18. Fiorio Pla A, Brossa A, Bernardini M, Genova T, Grolez G, Villers A, et al. Differential sensitivity of prostate tumor derived endothelial cells to sorafenib and sunitinib. BMC Cancer. 2014;14:939.

19. Avanzato D, Genova T, Fiorio Pla A, Bernardini M, Bianco S, Bussolati B, et al. Activation of P2X7 and P2Y11 purinergic receptors inhibits migration and normalizes tumor-derived endothelial cells via cAMP signaling. Sci Rep. 2016;6:32602.

20. Hida K, Kawamoto T, Ohga N, Akiyama K, Hida Y, Shindoh M. Altered angiogenesis in the tumor microenvironment. Pathol Int. 2011;61(11):630-7.

21. Hida K, Hida Y, Shindoh M. Understanding tumor endothelial cell abnormalities to develop ideal antiangiogenic therapies. Cancer Sci. 2008;99(3):459-66.

22. Genova T, Grolez GP, Camillo C, Bernardini M, Bokhobza A, Richard E, et al. TRPM8 inhibits endothelial cell migration via a non-channel function by trapping the small GTPase Rap1. J Cell Biol. 2017;216(7):2107-30.

23. Goel S, Duda DG, Xu L, Munn LL, Boucher Y, Fukumura D, et al. Normalization of the vasculature for treatment of cancer and other diseases. Physiol Rev. 2011;91(3):1071-121. 
24. Carmeliet P, Jain RK. Principles and mechanisms of vessel normalization for cancer and other angiogenic diseases. Nat Rev Drug Discov. 2011;10(6):417-27.

25. Cantelmo AR, Conradi LC, Brajic A, Goveia J, Kalucka J, Pircher A, et al. Inhibition of the Glycolytic Activator PFKFB3 in Endothelium Induces Tumor Vessel Normalization, Impairs Metastasis, and Improves Chemotherapy. Cancer Cell. 2016;30(6):968-85.

26. Carmeliet P. Means and methods for the treatment of pathological angiogenesis. \&\#x9;WO2013007766 A1. 2013.

27. Chesney J, Telang S, Yaddanapudi K. Combinations of pfkfb3 inhibitors and immune checkpoint inhibitors to treat cancer. WO2016191660 A1. Google Patents; 2016.

28. Patella F, Schug ZT, Persi E, Neilson L, Erami Z, Avanzato D, et al. Proteomics-based metabolic modeling reveals that fatty acid oxidation (FAO) controls endothelial cell (EC) permeability. Mol Cell Proteomics. 2015;14(3):621-34.

29. Carmeliet $P$, SCHOORS S. Carnitine palmitoyltransferase 1 inhibitors for inhibition of pathological angiogenesis. CA2918858 A1. 2015.

30. Carmeliet P, SCHOORS S. Carnitine palmitoyltransferase 1 inhibitors for inhibition of pathological angiogenesis. WO2015018660 A1. 2015.

31. Carmeliet P, SCHOORS S. Carnitine palmitoyltransferase 1 inhibitors for inhibition of pathological angiogenesis. EP3030240 A1. 2016.

32. Carmeliet P, Schoors S. Carnitine palmitoyltransferase 1 inhibitors for inhibition of pathological angiogenesis. US20160166532 A1. 2016.

33. Romero-Garcia S, Lopez-Gonzalez JS, Báez-Viveros JL, Aguilar-Cazares D, Prado-Garcia H. Tumor cell metabolism: an integral view. Cancer Biol Ther. 2011;12(11):939-48.

34. Hirschhaeuser F, Sattler UG, Mueller-Klieser W. Lactate: a metabolic key player in cancer. Cancer Res. 2011;71(22):6921-5.

35. Sonveaux P, Copetti T, De Saedeleer CJ, Végran F, Verrax J, Kennedy KM, et al. Targeting the lactate transporter MCT1 in endothelial cells inhibits lactate-induced HIF-1 activation and tumor angiogenesis. PLoS One. 2012;7(3):e33418.

36. Critchlow SE, Tate L. Use of a mct1 inhibitor in the treatment of cancers expressing mct1 over mct4. WO2010089580 A1. 2010.

37. Carmeliet P, EELEN G. Glutamine synthetase inhibitors for inhibition of pathological angiogenesis. WO2015018659 A1. 2015.

38. Mazzone M, Dettori D, de Oliveira RL, Loges S, Schmidt T, Jonckx B, et al. Heterozygous deficiency of PHD2 restores tumor oxygenation and inhibits metastasis via endothelial normalization. Cell. 2009;136(5):839-51.

39. De Bock K, De Smet F, Leite De Oliveira R, Anthonis K, Carmeliet P. Endothelial oxygen sensors regulate tumor vessel abnormalization by instructing phalanx endothelial cells. J Mol Med (Berl). 2009;87(6):561-9.

40. Carmeliet P, Mazzone M. Phd2 inhibition for blood vessel normalization, and uses thereof. CA2750044 A1. 2010.

41. Carmeliet P, Mazzone M. Phd2 inhibition for blood vessel normalization, and uses thereof. WO2010084134 A1. 2010.

42. Carmeliet P, Mazzone M. Phd2 inhibition for blood vessel normalization, and uses thereof. EP2389196 A1. 2011.

43. Carmeliet $\mathrm{P}$, Mazzone $\mathrm{M}$. Phd2 inhibition for blood vessel normalization, and uses thereof. US20120022135 A1. 2012.

44. Carmeliet P, Mazzone M, inventors; Google Patents, assignee. PHD2 inhibition for blood vessel normalization, and uses thereof2014.

45. Carmeliet P, Mazzone M. PHD2 inhibition for blood vessel normalization, and uses thereof. US8741862 B2. 2014.

46. Maes $\mathrm{H}$, Kuchnio A, Carmeliet $\mathrm{P}$, Agostinis $\mathrm{P}$. Chloroquine anticancer activity is mediated by autophagy-independent effects on the tumor vasculature. Mol Cell Oncol. 2016;3(1):e970097.

47. Maes $\mathrm{H}$, Kuchnio A, Peric A, Moens S, Nys K, De Bock K, et al. Tumor vessel normalization by chloroquine independent of autophagy. Cancer Cell. 2014;26(2):190-206. 
48. 卫立辛 吴, 宋建瑞, 李丁, 郭献灵. Pharmaceutical composition for cancer treatment. \&\#x9;CN101920015 A. 2010. 